\title{
Phenotypic Variability of Essential Tremor Based on the Age at Onset
}

\author{
Shweta Prasad, Ketaki Swapnil Bhalsing, Ketan Jhunjhunwala, Abhishek Lenka, \\ VS Binu, Pramod Kumar Pal
}

\begin{abstract}
Background: Essential tremor (ET) is reported to have a bimodal distribution of age at onset (AAO) with phenotypic variability based on the AAO. This study aims to explore the distribution of AAO based on mathematical modeling and ascertain the differences, if any, in the clinical features of groups. Methods: A chart review was conducted for 252 patients with ET diagnosed based on the Consensus statement of the Movement Disorder Society on Tremor. Finite mixture modeling was performed to identify groups of the cohort based on the AAO. Results: Three groups were defined: early onset (EO): AAO $\leq 22$ years, $n=63$, intermediate onset (IO): $23 \leq \mathrm{AAO} \leq 35$ years, $n=43$, and late onset (LO): AAO $\geq 36$ years, $n=146$. There were no significant differences related to family history or responsiveness to alcohol. The EO group had significantly higher prevalence of upper limb and lower limb tremor. Head tremor and voice tremor was more prevalent in the IO and LO groups. Cerebellar signs showed a significant increase with an increase in AAO. Conclusions: ET shows significant phenotypic variability based on the AAO. Patients with an early AAO are more likely to develop an appendicular tremor, whereas the probability of axial tremor and cerebellar signs increases with increasing AAO.
\end{abstract}

RÉSUMÉ: Variabilité phénotypique des tremblements essentiels en lien avec l'âge où la maladie est apparue. Contexte: Il semblerait que les tremblements essentiels (TE) sont caractérisés par une distribution bimodale de l'âge d'apparition (AA) de leurs premières manifestations et que la variabilité phénotypique de ces manifestations dépend de l'AA. Cette étude entend explorer la distribution de l'AA en se basant sur une modélisation mathématique. Le cas échéant, elle vise aussi à comprendre les variations entre les groupes de patients en ce qui regarde leurs caractéristiques cliniques. Méthodes: Nous avons ainsi passé en revue les dossiers de 252 patients chez qui l'on avait diagnostiqué des TE en se basant sur le Consensus statement of the Movement Disorder Society on Tremor. Nous avons ensuite effectué une modélisation par un mélange de distributions afin de déterminer des groupes formés en fonction de l'AA. Résultats: Trois groupes ont été définis : apparition précoce (AP) : AA $\leq 22$ ans, $\mathrm{n}=63$; apparition à un âge intermédiaire (AAI) : $23 \leq \mathrm{AA} \leq 35$ ans, $\mathrm{n}=43$; apparition tardive (AT) : AA $\geq 36$ ans, $\mathrm{n}=146$. Notons par ailleurs qu'aucune différence notable reliée à l'historique familiale des patients ou à leur réactivité à l'alcool n'a été observée. Cela dit, le groupe AP a donné à voir une prévalence nettement plus élevée de tremblements affectant les membres supérieurs et inférieures. Des tremblements affectant la tête et la voix se sont en revanche avérés nettement plus fréquents au sein des groupes AAI et AT. Enfin, mentionnons que les tremblements intentionnels (ou d'action) sont devenus nettement plus communs à mesure que l'AA des patients augmentait. Conclusions: Selon l'AA des TE, on a pu observer une variabilité phénotypique importante. Les patients du groupe AP sont ainsi plus susceptibles de développer des tremblements appendiculaires tandis que la probabilité de développer des tremblements axiaux et intentionnels s'accroît au fur et à mesure qu'augmente l'AA des TE.

Keywords: Essential tremor, Age at onset, Early onset, Late onset, Clinical features

doi:10.1017/cjn.2018.384

Can J Neurol Sci. 2019; 46: 192-198

\section{INTRODUCTION}

Essential tremor (ET) is the second most common movement disorder with an estimated prevalence between $0.4 \%$ and $3.9 \%{ }^{1}$ ET is classically associated with an action tremor of the upper limbs (ULs) and occasional involvement of the head, legs, and trunk. ${ }^{2}$ Additionally, a rest tremor and a variety of symptoms ranging from gait and balance impairments to alterations in cognition have also been reported. ${ }^{3-5}$
Epidemiological studies in ET have demonstrated a bimodal age at onset (AAO) with "early onset" that peaks in late 20 s and "late onset" with a peak in $50 \mathrm{~s} .6,7$ The possibility of clinical or pathophysiological differences between these groups has been repeatedly hypothesized but seldom explored. With the exception of a bilateral UL tremor, the remainder of clinical features in ET are highly heterogeneous and show significant variability based on AAO. Hopfner et al. ${ }^{8}$ reported variability in rates of

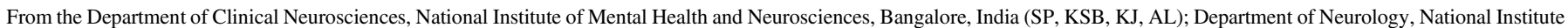

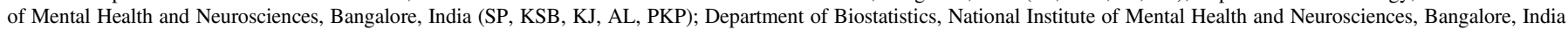
(VSB)

Received September 26, 2018. Final Revisions Submitted November 20, 2018. Date of Acceptance November $21,2018$.

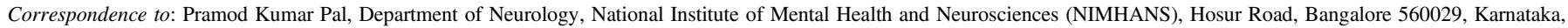
India. Email: palpramod@hotmail.com 
progression, frequencies of positive family history, and effect of alcohol on tremor, suggesting that early onset (EO) and late onset (LO) ET patients represent clinically distinct groups. However, similar postmortem cerebellar pathology in both groups refutes the possibility of pathological differences. ${ }^{9}$

The lack of coherence between clinical findings and neuropathological reports compounds the requirement for better classification and characterization of patients on the basis of AAO. This study aims to utilize mathematical modeling to explore the distribution of the AAO of patients with ET and ascertain differences, if any, in the clinical features of groups.

\section{Materials ANd Methods}

\section{Subject Recruitment and Clinical Evaluation}

This study was conducted at the Department of Neurology, National Institute of Mental Health and Neurosciences (NIMHANS), Bangalore, India. A chart review was conducted for patients with ET who were evaluated over a 5-year period at the neurology outpatient and movement disorder clinic. Some of the patients included in this study have been part of previous studies from our group. ${ }^{10,11}$ The diagnosis of ET was based on the Consensus statement of the Movement Disorder Society on Tremor $^{12}$ and confirmed by a trained movement disorder specialist. Demographic details such as gender, age at evaluation, AAO, presence of family history of tremor or parkinsonian illness, reduction of tremor after alcohol consumption, and chronology of body part involvement were recorded. Tremor of the ULs was assessed at rest, with arms outstretched, flexed at the elbow, and while performing the finger-nose test. Tremor of lower limbs (LLs) was assessed while lying in bed at the following positions - at rest, while the LL was lifted off the bed flexed $90^{\circ}$ at the hip and knee, while the LL was elevated at roughly $45^{\circ}$ with the knee extended, and while performing the knee-heel test. Head tremor (HT) was assessed with the patient seated and recorded as present or absent. Voice tremor (VT) was assessed during normal speech and during sustained phonation. Cerebellar signs were evaluated by the finger-nose test, kneeheel test, dysdiadochokinesia, and tandem gait. Patients also underwent a detailed neurological examination with special emphasis to rule out parkinsonism and dystonia. We also excluded the presence of thyrotoxicosis, chronic alcohol consumption, and the use of tremorogenic drugs.

\section{Statistical Analysis}

Statistical analysis was performed using $\mathrm{R}$ (version 3.4.3). ${ }^{13}$ Distribution of AAO was estimated using the following packages: diptest (v.0.75-7), mclust (v.5.4), and mixtools (v.1.1.0). ${ }^{14-16}$ Diptest was used to perform Hartigan's dip test in order to ascertain the basic distribution of the AAO dataset, that is, unimodal or bimodal. Following this, mclust was applied in order to identify statistically significant clusters in the dataset. Mclust performs finite mixture modeling for model-based clustering, classification, and density estimation and uses the Bayesian information criterion (BIC) to avoid overfitting. Finally, mixtools was used to graphically represent the results of mclust.

Descriptive statistical analysis was performed for the demographic features of patients with ET. Continuous variables were

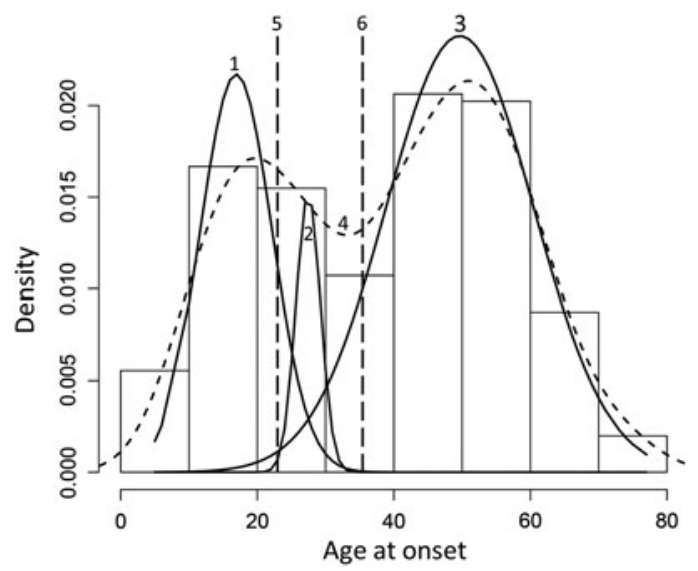

Figure 1: Histogram of the age at onset distribution with an overlay of the three sub distributions 1.EO, 2. IO, 3. LO, 4. Bimodal pattern of distribution observed on Hartigan's dip test, 5. Upper age limit of the EO group, 6. Lower age limit of the LO group.

analyzed using analysis of variance with post hoc Bonferroni correction, and the chi-square test was used for categorical variables.

To predict the presence of any significantly different variables with binary outcomes such as HT, cerebellar signs, etc., we performed multivariate binary logistic regression analysis. The covariates used for this analysis were AAO and a proxy for tremor severity (TSP). The TSP refers to a score ranging from 1 to 4 which indicates the number of sites with tremor involved in a single patient, with each site given a score of 1 . The sites considered were head, voice, ULs, and LLs, and the number of sites was chosen as a proxy for TSP since more sites tend to get involved as the disease progresses. The results of multivariate binary logistic regression were expressed as odds ratio (OR), confidence intervals (CIs), and $p$ value. Statistical significance was set at $p<0.05$.

\section{RESULTS}

\section{Demographic Data}

A total of 252 patients with ET were reviewed in this study. Hartigan's dip test revealed a bimodal distribution of AAO of the cohort $(p<0.05)$. The Gaussian finite mixture model fitted by Expectation-Maximization (EM) algorithm underlying normal distributions with unequal variance favored a three-component model over a two-component model (BIC: -2343.103 vs. -2373.52). The components of the three-component model were (a) Component 1: patients with EO ET (AAO: $15.57 \pm 4.28$ years), (b) Component 2: patients with intermediate onset (IO) ET (AAO: $28.40 \pm 3.60$ years), and (c) Component 3: patients with LO ET (AAO: $51.79 \pm 9.06$ years). We defined three groups based on two standard deviations as EO: AAO $\leq 22$, IO: $23 \leq \mathrm{AAO} \leq 35$, and LO: AAO $\geq 36$ (Figure 1). In addition to a comparison of the three groups - EO, IO, and LO - we also combined the EO and IO groups $(\mathrm{EO}+\mathrm{IO})$ and compared it with the LO group to ascertain if inclusion of the IO group with the EO group would alter the results.

\section{Comparison of EO, IO, and LO ET}

Men outnumbered women in all the three groups, and there were no significant gender differences between the three groups 
Table 1: Characteristics of patients with EO, IO, and LO ET

\begin{tabular}{l|c|c|c|c|c|c}
\hline & EO & IO & LO & EO vs. IO & IO vs. LO & EO vs. LO \\
\hline & $(\mathbf{A A O} \leq \mathbf{2 2})$ & $(\mathbf{2 3} \leq \mathbf{A A O} \leq \mathbf{3 5})$ & $(\mathbf{A A O} \geq \mathbf{3 6})$ & $\boldsymbol{p}$ value & $\boldsymbol{p}$ value & - \\
\hline Total number & 63 & 43 & 146 & - & Nalue \\
\hline Gender (M: F) & $44: 19$ & $28: 15$ & $99: 47$ & - & NS & - \\
\hline AAO (years) & $15.57 \pm 4.28$ & $28.40 \pm 3.60$ & $51.79 \pm 9.06$ & - & - & - \\
\hline $\begin{array}{l}\text { Age at presentation } \\
\text { (years) }\end{array}$ & $27.21 \pm 10.15$ & $38.88 \pm 10.88$ & $57.34 \pm 9.06$ & NS & - \\
\hline $\begin{array}{l}\text { Duration of illness } \\
\text { (years) }\end{array}$ & $11.25 \pm 11.30$ & $8.95 \pm 9.79$ & $5.36 \pm 4.12$ & NS & $<0.05$ & $<0.05$ \\
\hline Family history & $44.44 \%(28)$ & $55.8 \%(24)$ & $46.57 \%(68)$ & NS & NS & NS \\
\hline
\end{tabular}

$\mathrm{F}=$ female $\mathrm{M}=$ male; $\mathrm{NS}=$ not significant.

Table 2: Clinical features at the onset of illness and at evaluation

\begin{tabular}{|c|c|c|c|c|c|c|}
\hline & EO & IO & LO & EO vs. IO & IO vs. LO & EO vs. LO \\
\hline & $(\mathrm{AAO} \leq 22)$ & $(23 \leq \mathrm{AAO} \leq 35)$ & $(\mathrm{AAO} \geq 36)$ & $p$ value & $p$ value & $p$ value \\
\hline \multicolumn{7}{|l|}{ Onset of illness } \\
\hline HT & $2 \%(1)$ & $10 \%(6)$ & $21 \%(31)$ & NS & NS & $<0.01$ \\
\hline $\mathrm{U} / \mathrm{L} \mathrm{UL}$ & $29 \%(18)$ & $24 \%(15)$ & $38 \%(55)$ & NS & NS & NS \\
\hline $\mathrm{B} / \mathrm{L} \mathrm{UL}$ & $70 \%(44)$ & $35 \%(22)$ & $41 \%(60)$ & NS & NS & $<0.01$ \\
\hline \multicolumn{7}{|l|}{ At evaluation } \\
\hline \multicolumn{7}{|l|}{ Tremor Localization } \\
\hline HT & $0 \%(0)$ & $5 \%(2)$ & $8 \%(12)$ & NS & NS & $<0.01$ \\
\hline $\mathrm{B} / \mathrm{L} \mathrm{UL}$ & $73.01 \%(46)$ & $58.13 \%(25)$ & $53.42 \%(78)$ & NS & NS & $<0.01$ \\
\hline $\mathrm{B} / \mathrm{L} \mathrm{UL}+\mathrm{HT} \pm \mathrm{VT}$ & $14 \%(9)$ & $35 \%(15)$ & $34 \%(49)$ & $<0.01$ & NS & $<0.01$ \\
\hline $\mathrm{B} / \mathrm{L} \mathrm{UL}+\mathrm{B} / \mathrm{L} \mathrm{LL}$ & $13 \%(8)$ & $0 \%(0)$ & $3 \%(4)$ & $<0.05$ & $<0.05$ & $<0.01$ \\
\hline $\begin{array}{l}\mathrm{B} / \mathrm{L} \mathrm{UL}+\mathrm{B} / \mathrm{L} \mathrm{LL}+ \\
\mathrm{HT} \pm \mathrm{VT}\end{array}$ & $0 \%(0)$ & $2 \%(1)$ & $2 \%(3)$ & NS & NS & NS \\
\hline Cervical dystonia & $8 \%(5)$ & $7 \%(3)$ & $18 \%(26)$ & NS & NS & NS \\
\hline Cerebellar signs & $6 \%(4)$ & $23 \%(10)$ & $36 \%(52)$ & $<0.01$ & NS & $<0.01$ \\
\hline
\end{tabular}

$\mathrm{B} / \mathrm{L}=$ bilateral; $\mathrm{NS}=$ not significant; $\mathrm{U} / \mathrm{L}=$ unilateral.

(Table 1). The range of AAO was as follows: EO: 5-22 years, IO: 23-35 years, and LO: 36-73 years. Duration of illness was significantly different between the EO and LO, and IO and LO groups. There were no differences with regard to response of the tremor to alcohol or positive family history of ET.

\section{Tremor Topography}

Onset (Table 2): Tremor of bilateral ULs was the most common initial symptom across groups, and a significantly higher percentage was reported by the EO group in comparison with the LO group. A significantly higher percentage with LO reported HT as the symptom at onset in comparison with IO. Unilateral UL at onset was uniformly reported in all three groups.

At evaluation: Tremor localization at evaluation (Figure 2) revealed the uniform presence of UL tremor in all the three groups. The EO group had significantly higher prevalence of LL tremor in comparison with the LO group. The prevalence of HT

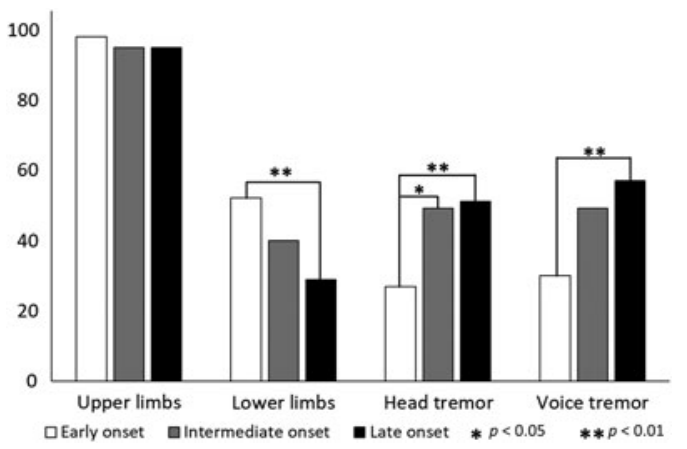

Figure 2: Topography of tremor at evaluation for $E O, I O$, and $L O$ groups. Equal prevalence of UL tremor in all the three groups. The EO group has a significantly higher prevalence of lower limb tremor in comparison with the LO group. The prevalence of HT significantly increases with age. VT was significantly higher in the LO group in comparison with the EO group. 
Table 3: Comparison of characteristics of patients with EO and IO ET versus LO ET

\begin{tabular}{l|c|c|c}
\hline & EO + IO & LO & EO+ IO vs. LO \\
\hline & (AAO $\leq \mathbf{3 5})$ & (AAO $\geq \mathbf{3 6})$ & $\boldsymbol{p}$ value \\
\hline Total number & 106 & 146 & - \\
\hline Gender (M: F) & $72: 34$ & $99: 47$ & NS \\
\hline AAO (years) & $20.77 \pm 7.45$ & $51.79 \pm 9.06$ & - \\
\hline Age at presentation (years) & $31.94 \pm 11.83$ & $57.34 \pm 9.06$ & - \\
\hline Duration of illness (years) & $10.32 \pm 10.67$ & $5.36 \pm 4.12$ & $p<0.01$ \\
\hline Family history & $49.05 \%(52)$ & $46.57 \%(68)$ & NS \\
\hline
\end{tabular}

$\mathrm{F}=$ female; $\mathrm{M}=$ male; $\mathrm{NS}=$ not significant.

Table 4: Comparison of clinical features at the onset of illness and at evaluation of patients with EO and IO ET versus LO ET

\begin{tabular}{|c|c|c|c|}
\hline & $\mathrm{EO}+\mathrm{IO}$ & LO & $\mathrm{EO}+\mathrm{IO}$ vs. $\mathrm{LO}$ \\
\hline & $(\mathbf{A A O} \leq 35)$ & $(\mathrm{AAO} \geq 36)$ & $p$ value \\
\hline HT & $7 \%(7)$ & $21 \%(31)$ & $<0.01$ \\
\hline $\mathrm{U} / \mathrm{L} \mathrm{UL}$ & $31 \%(33)$ & $38 \%(55)$ & NS \\
\hline $\mathrm{B} / \mathrm{L} \mathrm{UL}$ & $62 \%(66)$ & $41 \%(60)$ & $<0.01$ \\
\hline HT & $2 \%(2)$ & $8 \%(12)$ & $p<0.05$ \\
\hline $\mathrm{B} / \mathrm{L} \mathrm{UL}$ & $67 \%(71)$ & $53 \%(78)$ & $p<0.05$ \\
\hline $\mathrm{B} / \mathrm{L} \mathrm{UL}+\mathrm{HT} \pm \mathrm{VT}$ & $23 \%(24)$ & $34 \%(49)$ & NS \\
\hline $\mathrm{B} / \mathrm{L} \mathrm{UL}+\mathrm{B} / \mathrm{L} \mathrm{LL}$ & $8 \%(8)$ & $3 \%(4)$ & NS \\
\hline $\mathrm{B} / \mathrm{L} \mathrm{UL}+\mathrm{B} / \mathrm{L} \mathrm{LL}+\mathrm{HT} \pm \mathrm{VT}$ & $1.89 \%(2)$ & $2 \%(3)$ & NS \\
\hline Cervical dystonia & $8 \%(8)$ & $18 \%(26)$ & $p<0.05$ \\
\hline Cerebellar signs & $13 \%(14)$ & $36 \%(52)$ & $p<0.01$ \\
\hline
\end{tabular}

$\mathrm{B} / \mathrm{L}=$ bilateral; $\mathrm{NS}=$ not significant; $\mathrm{U} / \mathrm{L}=$ unilateral.

significantly increased with age, that is, the IO group had a significantly higher percentage of HT in comparison with the EO group, and the LO group had a significantly higher percentage of HT in comparison with the IO group. VT was also significantly higher in the LO group in comparison with the EO group.

On examination, patients displayed a variety of combinations of tremor localization (Table 2). An isolated HT was observed in $8 \%$ LO patients, which was significantly higher in comparison with the EO group, $p<0.01$. Bilateral UL tremor was the most common form of presentation and significantly higher in the EO group in comparison with the LO group. Bilateral UL tremor in combination with HT, with or without VT was significantly higher in the LO group in comparison with the EO group. Involvement of all four limbs was maximal in the EO group.

There were no significant differences observed based on the activation pattern of the UL tremor, that is, action or rest. An isolated action tremor was present in $71 \%, 72 \%$, and $62 \%$ of patients with EO, IO, and LO of ET. Both action and rest tremors were observed in $29 \%, 26 \%$, and $32 \%$ of patients with EO, IO, and LO of ET.

\section{Cervical Dystonia}

Mild cervical dystonia (CD) was observed in $13 \%(n=34)$ of subjects with ET, and no significant variability in prevalence was observed across groups (Table 2). Coexistence of CD and HT observed in $28 \%(n=32)$ subjects with a HT, and there were no significant differences in this prevalence across the groups.

\section{Cerebellar Signs}

The prevalence of cerebellar involvement in the form of impaired finger-nose test, knee-heel test, dysdiadochokinesia, or tandem walking was found to increase with the AAO. The LO group had a significantly higher prevalence of cerebellar signs in comparison with the EO group, $(36 \%$ vs. $6 \%, p<0.01)$. The prevalence of cerebellar signs in the IO group was also higher than the EO group $(23 \%$ vs. $6 \%, p<0.01)$.

None of the patients had features suggestive of dystonia or parkinsonism.

\section{Comparison of Early Plus IO ET with LO ET}

Men outnumbered women in both groups, and there were no significant gender differences between the two groups (Table 3). Duration of illness was significantly different between the EO + IO and LO groups. There were no differences with regard to response of the tremor to alcohol or positive family history of ET.

\section{Tremor Topography}

Onset (Table 4): The LO group had a higher prevalence of HT in comparison with $\mathrm{EO}+\mathrm{IO}$, and the $\mathrm{EO}+\mathrm{IO}$ group had a higher prevalence of bilateral UL tremor at the onset of disease. 


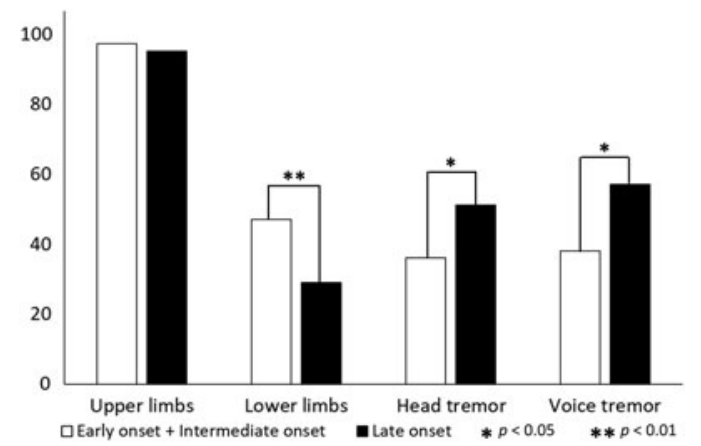

Figure 3: Topography of tremor at evaluation for $E O+I O$, and $L O$ groups Equal prevalence of UL tremor both groups. The EO + IO group has a significantly higher prevalence of lower limb tremor in comparison with the $L O$ group. The $L O$ had a significantly higher prevalence of HT and VT.

At evaluation: Tremor localization at evaluation (Figure 3) revealed the uniform presence of UL tremor in both groups. The $\mathrm{EO}+\mathrm{IO}$ group had significantly higher prevalence of LL tremor in comparison with the LO group. The prevalence of HT and VT was significantly higher in the LO group in comparison with the $\mathrm{EO}+\mathrm{IO}$ group.

On examination, patients displayed a variety of combinations of tremor localization (Table 4). An isolated HT was observed in $8 \%$ LO patients, which was significantly higher in comparison with the $\mathrm{EO}+\mathrm{IO}$ group. Isolated bilateral UL tremor was significantly more prevalent in the $\mathrm{EO}+\mathrm{IO}$ group. No significant differences were observed with other combinations of tremor localization.

There were no significant differences observed based on the activation pattern of the UL tremor, that is, action or rest. An isolated action tremor was present in $72 \%$ and $62 \%$ of patients with $\mathrm{EO}+\mathrm{IO}$ and LO of ET. Both action and rest tremors were observed in $27 \%$ and $32 \%$ of patients with $\mathrm{EO}+\mathrm{IO}$ and $\mathrm{LO}$ of ET.

\section{Cervical Dystonia}

A significantly higher prevalence of mild CD was observed in the LO group in comparison with $\mathrm{EO}+\mathrm{LO}$. There was no significant difference in the prevalence of coexistent $\mathrm{CD}$ and HT in the two groups.

\section{Cerebellar Signs}

The LO group had a significantly higher prevalence of cerebellar signs in comparison with the EO + IO group, $(36 \%$ vs. $13 \%, p<0.01)$.

\section{Multivariate Binary Logistic Regression}

Since the presence of HT and cerebellar signs was found to be significantly associated with an increasing AAO, we chose these two parameters to perform the regression analysis. AAO and TSP were significant predictors for the presence of HT, AAO (OR: 1.03; CI: 1.008-1.053; $p<0.01$ ), and TSP (OR: 11.59; CI: 6.486-20.724; $p<0.01)$. Only AAO was found to be a significant predictor for cerebellar signs (OR: 1.033; CI: 1.015-1.053, $p<0.01$ ).

\section{Discussion}

The AAO of ET has been reported to show a bimodal distribution with the possibility that EO and LO ET are clinically distinct groups. In the present study, mathematical modeling of the AAO of ET showed a trimodal distribution. We compared the phenotypic characteristics of 252 patients with ET, grouped as EO, IO, or LO based on the AAO.

Patients with LO of ET formed the largest group of our cohort. This finding is in concurrence with previous reports of the incidence and prevalence of ET increasing with age. ${ }^{17-19}$ Men outnumbered women in all three groups. This observation differs from community-based studies of ET from India which reported no gender discrepancy. ${ }^{20,21}$ It is plausible that the present observation is influenced by the nature of our cohort, that is, all patients being recruited from a tertiary care center. The LO group had a significantly shorter duration of illness prior to presentation which might indicate an increased severity of illness. Contrary to reports from other studies, the EO group in our cohort did not have a higher prevalence of family history of ET. ${ }^{7}$ However, the observed prevalence was in accordance with previous reports. ${ }^{7,22}$ It is possible that this result is secondary to a recall bias or tremor in an elderly relative being attributed to old age rather than ET. Although several studies have reported better alcohol responsiveness in patients with an EO of ET, we did not find similar results in our cohort. ${ }^{8,22}$ This could be due to a reporting bias or the social stigma associated with alcohol consumption. Additionally, several patients in the EO group presented for evaluation at an age lower than the legal drinking age, that is, 21 years.

Tremor localization at the time of onset and evaluation revealed significant differences. As expected, UL tremor was the predominant symptom across all groups. Isolated bilateral UL tremor was significantly higher in the EO group. This finding could either be due to a difference in the pathophysiology or more likely be due to patients in this group being more observant about the presence of a tremor in the hands, albeit a minimal tremor. The presence of a tremor involving all four limbs was maximal in the EO group. The above results could be suggestive of a higher appendicular involvement in patients with an early AAO of ET. Tremor localization at the onset of illness was based on history provided by patients. Even though ET is described as a symmetrical tremor, patients may report the presence of a unilateral tremor at onset with progression to a bilateral tremor.

HT at the onset of illness was significantly higher in the LO group. This finding has been reported by several previous studies. ${ }^{11,23}$ Louis $^{23}$ had suggested that the appearance of a HT in ET was secondary to an intrinsic biological factor, that is, age or aging rather than the disease duration. This concept is supported by the increasing trend of HT on examination which was observed in our cohort. This trend was found to be inversely proportional to the duration of illness, that is, IO and LO groups had shorter duration of illness and a higher prevalence HT in comparison with the EO group. The prevalence of a VT in ET ranges from $10 \%$ to $62 \% .{ }^{24} \mathrm{~A}$ trend similar to the HT was also observed for a VT. However, it is uncertain whether this tremor was secondary to the HT influencing the vocal air stream and imparting a tremulous quality to the voice or due to the underlying pathophysiology of ET. The HT observed in ET is often considered to be dystonic, and mild $\mathrm{CD}$ has been frequently 
observed in patients with ET. ${ }^{11}$ We observed this in $13 \%$ of subjects in this cohort with a higher prevalence of $\mathrm{CD}$ in the LO group. Additionally, although $94 \%$ of subjects with CD had a HT, only $28 \%$ of subjects with HT had CD, and no significant variations in prevalence were observed between groups.

No differences were observed in the activation patterns of tremor across groups. An isolated action tremor or a combination of action and rest tremor was equally observed. The prevalence of rest tremor in ET ranges from $1.9 \%$ to $46.4 \%$ and has been reported to be associated with older age and longer disease duration. ${ }^{25}$

The increasing trend of cerebellar signs observed with an increase in AAO is highly suggestive of a difference in the degree of cerebellar involvement with AAO. The presence of this observation despite a shorter duration of illness in the IO and LO groups in comparison with the EO group further supports this concept. An increase in the prevalence of cerebellar signs in older patients of ET has been previously reported. ${ }^{26}$ The presence of cerebellar signs and $\mathrm{CD}$ in ET is of importance following the introduction of the category "ET plus syndrome." 2 The ET plus syndrome includes patients with ET who demonstrate neurological signs of uncertain significance such as mild cerebellar signs, dystonic posturing, rest tremor, etc. in addition to the classical tremor. Future studies classifying patients as ET and ET plus are necessary to study the underlying basis for these seemingly similar disorders.

Based on the pattern of tremor topography, it may be postulated that patients with LO of ET have significantly higher axial involvement, while the EO group has appendicular involvement. The significant variations in the prevalence of cerebellar signs may be indicative of differences in underlying disease pathophysiology. Although, the study by Kuo et al. ${ }^{9}$ did not demonstrate any differences in cerebellar pathology, further studies are necessary to validate this finding. The significant predictive value of AAO for HT and cerebellar signs obtained from regression analysis lends support to the role of AAO in the development of these symptoms.

Hopfner et al. ${ }^{8}$ followed a similar methodology when evaluating the possibility of clinical groups in ET, and despite the modeling suggesting a trimodal distribution, they evaluated only the EO and LO groups. We chose to compare all three groups in order to ascertain if the IO had different characteristics which could be indirectly indicative of the differences in the pathophysiology as the AAO increases. The lack of a clear pattern of topography in the IO group may be suggestive of the IO group being a transition group or a third clinical group of ET, which may be found to have specific characteristics if explored in a larger cohort. Several significant differences observed when three groups were compared were lost when the EO and IO group were combined, supporting the possibility of differences between these three groups.

This study has several limitations owing to its retrospective nature. Details pertaining to AAO and symptom at onset were based on history provided by the patient, hence subject to recall bias. We did not apply TSP rating scales to patients in our cohort, due to which we were unable to quantify the disease severity in groups and correlate it with variables such as duration of illness and age at evaluation. Additionally, the treatment profile and response to medication were not evaluated.

\section{Conclusions}

ET shows phenotypic variability based on the AAO. Patients with an EO of tremor are more likely to develop an appendicular tremor. Whereas, the probability of developing an axial tremor and cerebellar signs increases as the AAO increases. Further studies focusing on ascertaining the clinical, radiological, or pathological characteristics of patients with ET based on their AAO are necessary to enable a better understanding of this disease.

\section{Disclosure}

None of the authors has any financial disclosure to make or any conflict of interest.

\section{Author Roles}

Conception and design of study: SP, VSB, PKP.

Acquisition of data: SP, KSB, KJ, AL.

Analysis/interpretation of data: SP, VSB, PKP.

Drafting the manuscript: SP, PKP.

Revising the manuscript critically: SP, PKP.

\section{REFERENCES}

1. Louis ED, Ferreira JJ. How common is the most common adult movement disorder? Update on the worldwide prevalence of essential tremor. Mov Disord. 2010;25(5):534-41.

2. Bhatia KP, Bain P, Bajaj N, et al. Consensus Statement on the classification of tremors, from the task force on tremor of the International Parkinson and Movement Disorder Society. Mov Disord. 2018;33(1):75-87.

3. Chandran V, Pal PK. Essential tremor: beyond the motor features. Parkinsonism Relat Disord. 2012;18(5):407-13.

4. Bermejo-Pareja F. Essential tremor - a neurodegenerative disorder associated with cognitive defects? Nat Rev Neurol. 2011;7(5): 273-82.

5. Prasad S, Velayutham SG, Reddam VR, Stezin A, Jhunjhunwala K, Pal PK. Shaky and unsteady: dynamic posturography in essential tremor. J Neurol Sci. 2018;385:12-6.

6. Louis ED, Ottman R. Study of possible factors associated with age of onset in essential tremor. Mov Disord. 2006;21(11):1980-6.

7. Louis ED, Dogu O. Does age of onset in essential tremor have a bimodal distribution? Data from a tertiary referral setting and a population-based study. Neuroepidemiology. 2007;29(3-4): 208-12.

8. Hopfner F, Ahlf A, Lorenz D, et al. Early- and late-onset essential tremor patients represent clinically distinct subgroups. Mov Disord. 2016;31(10):1560-6.

9. Kuo SH, Wang J, Tate WJ, et al. Cerebellar pathology in early onset and late onset essential tremor. Cerebellum. 2017;16(2): 473-82.

10. Bhalsing KS, Kumar KJ, Saini J, Yadav R, Gupta AK, Pal PK. White matter correlates of cognitive impairment in essential tremor. AJNR Am J Neuroradiol. 2015;36(3):448-53.

11. Lenka A, Bhalsing KS, Jhunjhunwala KR, Chandran V, Pal PK. Are patients with limb and head tremor a clinically distinct subtype of essential tremor? Can J Neurol Sci. 2015;42(3):181-6.

12. Deuschl G, Bain P, Brin M, Ad Hoc Scientific Committee. Consensus statement of the movement disorder society on tremor. Mov Disord. 1998;13(Suppl. 3):2-23.

13. Team RC. R: a language and environment for statistical computing. Vienna, Austria: R Foundation for Statistical Computing; 2014.

14. Maechler M, Ringach D. Diptest: Hartigan's dip test statistic for unimodality-corrected code. R package version 025-2; 2009.

15. Benaglia T, Chauveau D, Hunter D, Young D. mixtools: an $R$ package for analyzing finite mixture models. J Stat Software. 2009;32(6):1-29.

16. Fraley C, Raftery AE. Model-based clustering, discriminant analysis, and density estimation. J Am Stat Assoc. 2002;97(458): 611-31.

17. Benito-Leon J, Bermejo-Pareja F, Louis ED, Neurological Disorders in Central Spain Study G. Incidence of essential tremor in three 
elderly populations of central Spain. Neurology. 2005;64(10): 1721-5.

18. Benito-Leon J, Bermejo-Pareja F, Morales JM, Vega S, Molina JA. Prevalence of essential tremor in three elderly populations of central Spain. Mov Disord. 2003;18(4):389-94.

19. Rajput AH, Offord KP, Beard CM, Kurland LT. Essential tremor in Rochester, Minnesota: a 45-year study. J Neurol Neurosurg Psychiatry. 1984;47(5):466-70.

20. Bharucha NE, Bharucha EP, Bharucha AE, Bhise AV, Schoenberg BS. Prevalence of essential tremor in the Parsi community of Bombay, India. Arch Neurol. 1988;45(8):907-8.

21. Das SK, Banerjee TK, Roy T, Raut DK, Chaudhuri A, Hazra A. Prevalence of essential tremor in the city of Kolkata, India: a house-to-house survey. Eur J Neurol. 2009;16(7):801-7.
22. Lou JS, Jankovic J. Essential tremor: clinical correlates in 350 patients. Neurology. 1991;41(2 (Pt 1)):234-8.

23. Louis ED. When do essential tremor patients develop head tremor? Influences of age and duration and evidence of a biological clock. Neuroepidemiology. 2013;41(2):110-5.

24. Sulica L, Louis ED. Clinical characteristics of essential voice tremor: a study of 34 cases. Laryngoscope. 2010;120(3):516-28.

25. Louis ED, Hernandez N, Michalec M. Prevalence and correlates of rest tremor in essential tremor: cross-sectional survey of 831 patients across four distinct cohorts. Eur J Neurol. 2015; 22(6):927-32.

26. Benito-Leon J, Labiano-Fontcuberta A. Linking essential tremor to the cerebellum: clinical evidence. Cerebellum. 2016;15(3): 253-62. 